TRANSACTIONS OF THE

AMERICAN MATHEMATICAL SOCIETY

Volume 359, Number 9, September 2007, Pages 4073-4087

S 0002-9947(07)04397-8

Article electronically published on April 11, 2007

\title{
GEOMETRY OF PHASE SPACE AND SOLUTIONS OF SEMILINEAR ELLIPTIC EQUATIONS IN A BALL
}

\author{
JEAN DOLBEAULT AND ISABEL FLORES
}

Abstract. We consider the problem

$$
\left\{\begin{array}{l}
-\Delta u=u^{p}+\lambda u \quad \text { in } B, \\
u>0 \text { in } B, \quad u=0 \text { on } \partial B,
\end{array}\right.
$$

where $B$ denotes the unit ball in $\mathbb{R}^{N}, N \geq 3, \lambda>0$ and $p>1$. Merle and Peletier showed that for $p>\frac{N+2}{N-2}$ there is a unique value $\lambda=\lambda_{*}>0$ such that a radial singular solution exists. This value is the only one at which an unbounded sequence of classical solutions of (1) may accumulate. Here we prove that if additionally

$$
p<\frac{N-2 \sqrt{N-1}}{N-2 \sqrt{N-1}-4} \text { or } \quad N \leq 10,
$$

then for $\lambda$ close to $\lambda_{*}$, a large number of classical solutions of (1) exist. In particular infinitely many solutions are present if $\lambda=\lambda_{*}$. We establish a similar assertion for the problem

$$
\left\{\begin{array}{l}
-\Delta u=\lambda f(u+1) \text { in } B, \\
u>0 \text { in } B, \quad u=0 \text { on } \partial B,
\end{array}\right.
$$

where $f(s)=s^{p}+s^{q}, 1<q<p$, and $p$ satisfies the same condition as above.

\section{INTRODUCTION}

Let $B$ denote the unit ball in $\mathbb{R}^{N}, N \geq 3$, and consider bounded solutions of

$$
\left\{\begin{array}{l}
-\Delta u=u^{p}+\lambda u \text { in } B, \\
u>0 \text { in } B, \quad u=0 \text { on } \partial B .
\end{array}\right.
$$

According to 17, classical solutions of this problem need to be radially symmetric and decreasing, so that with a straightforward abuse of notations, we may write $u=u(r), r=|x|$, and reduce the existence problem to that of the two-point boundary value problem

$$
\left\{\begin{array}{l}
u^{\prime \prime}+\frac{N-1}{r} u^{\prime}+u^{p}+\lambda u=0, \quad 0<r<1, \\
u^{\prime}(0)=0, \quad u(1)=0 \text { and } u(r)>0 \text { for } 0<r<1 .
\end{array}\right.
$$

If the equation $\Delta u+u^{p}+\lambda u=0$ is written in a general bounded smooth domain $\Omega$ and supplied with zero Dirichlet boundary conditions as in (1), then for any value

Received by the editors March 24, 2004.

2000 Mathematics Subject Classification. Primary 35B33; Secondary 34C37, 34C20, 35J60.

(C) 2007 American Mathematical Society Reverts to public domain 28 years from publication 
of the exponent $p$, no solution exists for $\lambda \geq \lambda_{1}$, where $\lambda_{1}$ is the first eigenvalue of $-\Delta$ under zero Dirichlet boundary conditions in $\Omega$. This is easily recovered by multiplying the equation by the first eigenfunction and performing an integration by parts. On the other hand, if $\lambda<\lambda_{1}$, the existence of a positive solution holds for any $p<\frac{N+2}{N-2}$, as it follows from a standard constrained minimization procedure involving compactness of the Sobolev embeddings. Now, by Pohozaev's identity [29, there are no solutions for $\lambda \leq 0$ whenever $p \geq \frac{N+2}{N-2}$ if $\Omega$ is star-shaped, and, in particular, if it is a ball.

In 6] Brezis and Nirenberg established that this problem for $p=\frac{N+2}{N-2}$ is solvable for $\underline{\lambda}<\lambda<\lambda_{1}$, where $\underline{\lambda}=0$ for $N \geq 4$ and $0<\underline{\lambda}<\lambda_{1}$ if $N=3$. In the case of a ball, they explicitly found that $\underline{\lambda}=\lambda_{1} / 4$. These results can be described in terms of the branch of positive solutions of (1). From standard bifurcation theory, the set of pairs $(\lambda, u(0))$ where $u$ solves (11) is a curve which stems from $\lambda=\lambda_{1}$ and $u=0$. In [25, 33, 34, 19, 32, 15], it is shown that, if $p \leq \frac{N+2}{N-2}$, there is at most one solution of (11) for $\lambda<\lambda_{1}$ (also see [14, 31, 26, 27, 30] for more recent results). Hence in this case the curve goes left, without turning points, and if $p=\frac{N+2}{N-2}$ it blows up as $\lambda \downarrow \underline{\lambda}$.

The situation drastically changes as soon as the critical exponent is crossed. Budd and Norbury in [7] considered (1) for $p>\frac{N+2}{N-2}$, and formally derived qualitative properties of this bifurcation branch. In particular, formal asymptotics and numerical computations suggest that the following takes place: Before reaching $\lambda=0$, the curve turns right and then oscillates infinitely many times in the form of an exponentially damped sinusoidal along a line $\lambda=\lambda_{*}$. Merle and Peletier in 23] rigorously established for $N \geq 4$ the existence of this unique $\lambda=\lambda_{*}>0$ for which necessarily $\lambda_{n} \rightarrow \lambda_{*}$ whenever $u_{n}$ is an unbounded sequence of classical solutions of (11) for $\lambda=\lambda_{n}$. Moreover, at $\lambda=\lambda_{*}$, and only for this value of $\lambda$, a unique singular radial solution exists (see also [24]).

In this paper we rigorously establish the validity of the picture suggested by Budd and Norbury [7] in the sense that a large number of solutions of (1) exists for $\lambda$ close to $\lambda_{*}$. In fact, infinitely many classical solutions exist at $\lambda=\lambda_{*}$ provided that $p$ is supercritical but not too large.

Theorem 1. Assume that $N \geq 3, p>\frac{N+2}{N-2}$. Suppose moreover that

$$
\text { either } \quad p<\frac{N-2 \sqrt{N-1}}{N-2 \sqrt{N-1}-4} \quad \text { or } \quad N \leq 10 \text {. }
$$

Then there is a unique number $\lambda_{*}>0$ such that, given any integer $k \geq 1$, there exist at least $k$ bounded radial solutions of (11) for any $\lambda$ sufficiently close to $\lambda_{*}$. In particular, there are infinitely many classical solutions of (11) for $\lambda=\lambda_{*}$.

A question that has attracted the interest of various authors is that of the existence of sign-changing solutions when the nonlinearity $u^{p}$ is replaced by its odd extension $|u|^{p-1} u$, namely

$$
\left\{\begin{aligned}
-\Delta u=|u|^{p-1} u+\lambda u & \text { in } \quad B \\
u=0 & \text { on } \partial B .
\end{aligned}\right.
$$

Starting from the $n$-th radial eigenvalue $\lambda_{n}$ of the Laplacian under zero Dirichlet boundary condition, a branch of radial solutions with exactly $n-1$ zeros bifurcates from $\left(\lambda_{n}, 0\right)$. For the critical exponent $p=\frac{N+2}{N-2}$, the asymptotic behavior of this 
branch has been analyzed in [9] for $N \geq 7$ and in 1 for $4 \leq N \leq 6$. Roughly speaking, the $n$-th branch lives in the range $\lambda \in\left(0, \lambda_{n}\right)$ if $N \geq 7$, while it blows-up above $\lambda=0$ if $N \leq 6, n \geq 2$. In the super-critical case, results on classification of singular and classical solutions have been recently found in [5, 13. Our second result can be visualized by saying that the $n$-th branch develops at some number $\lambda=\lambda_{n}^{*}$ a behavior which is similar to that predicted in Theorem 1 for positive solutions.

Theorem 2. Assume that $N \geq 3$, and that $p$ satisfies the constraints of Theorem 1. Then there exists a strictly increasing sequence of positive numbers $\left(\lambda_{n}^{*}\right)_{n \geq 1}$ such that the following holds. Given any integer $k \geq 1$, there exist at least $k$ radial classical solutions of (3) with exactly $n-1$ zeros for any $\lambda$ sufficiently close to $\lambda_{*}$. In particular, there are infinitely many solutions of (3) for $\lambda=\lambda_{n}^{*}$. Besides, a unique singular solution with $n-1$ zeros, which is positive near 0 , exists for this value of $\lambda$, and there is no other one with the same properties for other values of $\lambda$.

The proof of Theorem 2 is an easy extension of the proof of Theorem 1 Closely related with (11) is the problem

$$
\left\{\begin{array}{l}
-\Delta u=\lambda(u+1)^{p} \text { in } B, \\
u>0 \text { in } B, \quad u=0 \text { on } \partial B,
\end{array}\right.
$$

with $\lambda>0$. This problem has been considered by Joseph and Lundgren in [22. In this paper, they completely describe the branch $(\lambda, u)$ of positive radial solutions, which in this case stems from $(0,0)$. For instance, if $p$ satisfies the constraints of Theorem 1, it is rigorously shown that this branch turns left at some positive value of $\lambda$ and then oscillates around some number $\lambda=\lambda_{*}$, at which the same conclusions as the ones of Theorem 1 hold. The main difference between the analysis of problem (11) and that in 22 of (44) is the fact that in the latter, the associated ODE can be reduced via a suitable change of variables to an autonomous second order ODE. This problem may then be fully analyzed via a phase plane analysis. Recently in 20 , the case of $p$-Laplacian and of $k$-Hessian operators, where the autonomous reduction is still possible, has been analyzed when the right-hand side in (4) is replaced by $\lambda e^{u}$. For $p=2$, this case was also treated in [22], and similar phenomena were observed for $3 \leq N \leq 9$. Although it looks similar, problem (1) is considerably more delicate since such a reduction is apparently not possible. In fact it is not clear whether the result in 22 holds true if the power $s^{p}$ is perturbed by lower order terms. To exemplify the robustness of the method we employ here, we consider the problem

$$
\left\{\begin{array}{l}
-\Delta u=\lambda f(u+1) \text { in } B, \\
u>0 \text { in } B, \quad u=0 \text { on } \partial B .
\end{array}\right.
$$

Theorem 3. Let $f(s)=s^{p}+s^{q}$ in problem (5) where $1<q<p$. If $p>\frac{N+2}{N-2}$ and

$$
\text { either } p<\frac{N-2 \sqrt{N-1}}{N-2 \sqrt{N-1}-4} \quad \text { or } \quad N \leq 10 \text {, }
$$

then there is a number $\lambda_{*}>0$ such that given any integer $k \geq 1$, there exist at least $k$ classical solutions of (11) for any $\lambda$ sufficiently close to $\lambda_{*}$. 
The proofs of Theorems 12 and 3 consist in performing a suitable transformation of the problem, the so-called Emden-Fowler transformation [16], also used in [22]. This leads to a second order ODE which is not autonomous. The results follow after a topological analysis of the situation arising in the three-dimensional phase space using tools of the geometric theory of dynamical systems. Although in a slightly different context, phase space analysis has been the issue in several related works on radial solutions to semilinear elliptic equations; see for instance [5, 2, 8, 21. Also note that a variational analysis can be done in the slightly supercritical case, i.e. for $p-\frac{N+2}{N-2}>0$, small [10, 12, which also has an ODE interpretation [11.

\section{Preliminaries: The Set-up}

We search for positive radially symmetric solutions $u=u(r), r=|x|$, of (1), namely solutions of the ordinary differential equation (2). Consider the classical Emden-Fowler transformation

$$
x(t)=\left.r^{\frac{2}{p-1}} u(r)\right|_{r=e^{t}},
$$

which recasts (2) into the equivalent problem

$$
x^{\prime \prime}+\alpha x^{\prime}+|x|^{p-1} x+e^{2 t} \lambda x-\beta x=0, \quad-\infty<t<0,
$$

where

$$
\alpha:=(N-2)-\frac{4}{p-1} \quad \text { and } \quad \beta:=\frac{2}{p-1}\left(N-2-\frac{2}{p-1}\right) .
$$

Straightforward calculations show that finding a positive solution of (2) is equivalent to finding a solution $x(t)$ of (7) such that

$$
x(t)>0 \quad \text { in } \quad(-\infty, 0), \quad \lim _{t \rightarrow-\infty} x(t)=0 \quad \text { and } \quad x(0)=0 .
$$

Now, we introduce the variables $y=x^{\prime}$ and $z=e^{2 t} \lambda$. In these variables, (7) becomes equivalent to the autonomous first order system

$$
\left\{\begin{array}{l}
x^{\prime}=y, \\
y^{\prime}=-\alpha y+\beta x-|x|^{p-1} x-z x, \\
z^{\prime}=2 z
\end{array}\right.
$$

with the additional constraint $z \geq 0$. At this level, it turns out that finding a solution $x(t)$ of (7)-(8) is equivalent to finding a solution $\varphi(t)=(x(t), y(t), z(t))$ of (9) such that

$$
x(t)>0 \quad \text { in } \quad(-\infty, 0), \quad \lim _{t \rightarrow-\infty} \varphi(t)=(0,0,0) \quad \text { and } \quad x(0)=0 .
$$

The rest of this section will be devoted to the phase space analysis of system (9). First we describe the general structure of the orbits and introduce some notation which will be useful in the subsequent analysis. We start with some straightforward facts for the flow in the supercritical case $p>\frac{N+2}{N-2}$ :

(i) The positive $z$-axis is an orbit for the system.

(ii) The plane $\{z=0\}$ is invariant under the flow of (9). It is in correspondence with (2) with $\lambda=0$ after the Emden-Fowler transformation.

(iii) System (9) is invariant under $(x, y, z) \mapsto(-x,-y, z)$, so that in what follows, we will concentrate our analysis on $x \geq 0$. 
(iv) Orbits that intersect the plane $\{x=0\}$ do it transversally. For $y>0$, they go into the region $x>0$, while the opposite happens for $y<0$.

(v) The two-dimensional foliation $\Sigma$ given by

$$
\Sigma:\left\{\left\{(x, y, z) \in \mathbb{R}^{3}: z=c\right\}, \quad c \in \mathbb{R}_{+}\right\}
$$

is invariant under the flow. Moreover, the planes $\{z=c\}$ move upwards in time; in fact $z(t)=z_{0} e^{\gamma t}$ with $\gamma$ positive.

(vi) The system has exactly three singularities,

$O=(0,0,0), \quad P=\left(\beta^{1 /(p-1)}, 0,0\right) \quad$ and $\quad P_{-}=\left(-\beta^{1 /(p-1)}, 0,0\right)$,

which are hyperbolic saddle points. At each of these three points, there are well-defined stable and unstable manifolds associated to these singularities.

Linearizing (9) around $O$ we obtain two unstable eigenvalues $2 /(p-1)$ and 2 with associated eigenvectors $(1,2 /(p-1), 0)$ and $(0,0,1)$, and one stable eigenvalue $2 /(p-1)-(N-2)$ with eigenvector $(1,2 /(p-1)-(N-2), 0)$. Thus, from standard invariant manifold theory (see for instance [18), $O$ has a two-dimensional unstable manifold $W^{u}(O)$, constituted by all trajectories approaching $O$ as $t \rightarrow-\infty$, whose tangent plane is spanned by the two unstable eigenvectors. The manifold $W^{u}(O)$ contains the entire $z$-axis as well as the heteroclinic orbit in $\{z=0\}$ connecting $O$ and $P$. It is also transversal to the planes $\{z=0\}$ and $\{x=0\}$; see Figure 1 in section 6 .

Linearizing around the singularity $P$, we obtain one unstable eigenvalue, 2 , with an associated eigenvector

$$
\left(1,2, \frac{(p-1) \beta+2 \alpha+4}{\beta^{1 /(p-1)}}\right)
$$

and two stable eigenvalues

$$
\frac{1}{2}\left(-\alpha \pm \sqrt{\alpha^{2}-4 \beta(p-1)}\right) .
$$

By a direct computation we see that these eigenvalues are complex if and only if relation

$$
p<\frac{N-2 \sqrt{N-1}}{N-2 \sqrt{N-1}-4} \quad \text { or } \quad N \leq 10
$$

holds. Thus, the unstable manifold of $P$ for $z \geq 0, W^{u}(P)$, is one-dimensional, and constituted by a single orbit. Its stable manifold is two-dimensional and lies in the plane $\{z=0\}$; see Figure 2 in section 6 .

Lemma 1. The unstable manifold of $P, W^{u}(P)$, is contained in the closure of the unstable manifold of $O, W^{u}(O)$.

We postpone the proof of this fact until the Appendix. We shall say that two systems $x^{\prime}=f(x)$ and $y^{\prime}=g(y)$ with respective singularities $P$ and $Q$ are $C^{1}$ equivalent around these points if there is a $C^{1}$-diffeomorphism between neighborhoods of these points which transforms trajectories of the first system into trajectories of the other one, preserving orientations. The following fact will be important for our purposes.

Lemma 2. System (9) is $C^{1}$-equivalent to its linearized system around $P$, provided that condition (11) holds.

We also leave this result until the Appendix and go to the proof of Theorem 1 


\section{Proof of Theorem 1}

We look for positive solutions to (2). At the level of the orbit $x$ given by (6), this means that $(x(t), y(t), z(t))$ is attracted by $O$ as $t \rightarrow-\infty$, namely that it lies in $W^{u}(O)$ and $x$ remains positive for $t<0$ and $x=0$ for $t=0$.

We define $\lambda_{*}$ to be the height $z=\lambda_{*}$, where $W^{u}(P)$ first intersects the plane $\{x=0\}$. This intersection is transversal because of the uniqueness of the solution to the initial value problem for the second order equation (7). Besides, let us observe that a trajectory associated to the one-dimensional manifold $W^{u}(P)$ is in exact correspondence with the unique positive singular solution to (2), which exists if and only if $\lambda=\lambda^{*}$; see 23 .

Hence our task is to show that under the conditions of Theorem 1 there are infinitely many distinct trajectories lying in the two-dimensional manifold $W^{u}(O)$ which remain positive in their $x$-coordinate for $z<\lambda_{*}$ and are such that for $z=$ $\lambda_{*}$ their $x$-coordinate is 0 . The strategy is as follows: we prove that the curve corresponding to $W^{u}(O) \cap\left\{z=\lambda_{*}\right\}$ is a spiral curve which winds around the point $W^{u}(P) \cap\left\{z=\lambda_{*}\right\}$. This fact gives infinitely many intersections of $W^{u}(O) \cap\left\{z=\lambda_{*}\right\}$ and the plane $\{x=0\}$.

From Lemma 2, we know that system (9) is $C^{1}$-equivalent in a neighborhood of $P$ to the following linear system:

$$
\left\{\begin{array}{l}
\bar{x}^{\prime}=\bar{y}, \\
\bar{y}^{\prime}=a \bar{x}+b \bar{y}+c \bar{z}, \\
\bar{z}^{\prime}=2 \bar{z},
\end{array}\right.
$$

where $a=\beta(1-p), b=-\alpha, c=-\beta^{1 /(p-1)}$. After a suitable linear transformation, we immediately check that this system is also $C^{1}$-equivalent to

$$
\left\{\begin{array}{l}
\bar{x}^{\prime}=\bar{y}, \\
\bar{y}^{\prime}=a \bar{x}+b \bar{y}, \\
\bar{z}^{\prime}=2 \tilde{z} .
\end{array}\right.
$$

Let $\Phi: \mathcal{V} \rightarrow \mathcal{U}$ be the diffeomorphism setting the equivalence between (9) and (12), where $\mathcal{V}$ is a neighborhood of $P$ and $\mathcal{U}$ is one of $\bar{O}=(0,0,0)$. As in the original system (9), the origin in (12) is an attractor focus when (12) is restricted to the plane $\{\bar{z}=0\}$ and the $\bar{z}$-axis is its corresponding unstable manifold.

Let us recall that according to Lemma 1 the unstable manifold of $O$ contains the unstable manifold of $P$ in its closure. Hence in the neighborhood $\mathcal{V}$ of $P$ where the equivalence is valid, there is an invariant manifold, namely $W^{u}(O) \cap \mathcal{V}$. To this manifold, there corresponds through the equivalence $\Phi$ an invariant manifold $\mathcal{M}$ of system (12) inside $\mathcal{U}$. Let $\{\psi(t): t \in \mathbb{R}\}$ be the image through $\Phi$ restricted to $\{z=0\} \cap \mathcal{V}$ of the orbit corresponding to the heteroclinic trajectory which connects $O$ and $P$. It is easily checked, after making $\mathcal{U}$ and $\mathcal{V}$ smaller if necessary, that the manifold $\mathcal{M}$ must be constituted exactly by the set of points of the form $\{(\psi(t), \bar{z}): t \in \mathbb{R}, \bar{z} \in \mathbb{R}\}$ which lie inside $\mathcal{U}$, the neighborhood of $O$. See Figure 3 in section 6 for more details.

Let us consider a small $z_{0}$ so that the plane $\left\{z=z_{0}\right\}$ intersects $\mathcal{V}$. Let us also consider the image of $W^{u}(O) \cap\left\{z=z_{0}\right\}$ through the equivalence $\Phi$. The image of this curve through $\Phi$ is a curve which lies on $\mathcal{M}$. Since $\mathcal{M}$ is given by the set $\{(\psi(t), \bar{z}): t \in \mathbb{R}, \bar{z} \in \mathbb{R}\} \cap \mathcal{U}$ inside $\mathcal{U}$, and $\Phi$ is a $C^{1}$-diffeomorphism, then this curve is a spiral curve which lies inside the image by $\Phi$ of the plane $\left\{z=z_{0}\right\}$, which 
is surface transversal to the flow associated to system (12), and $C^{1}$-close to the plane $\left\{\bar{z}=\Phi\left(z_{0}\right)\right\}$ in an appropriately small neighborhood. See Figure 4 in section 6. This proves that $W^{u}(O) \cap\left\{z=z_{0}\right\}$ is a spiral curve. Now, by the transition map, we can prove that for all $z_{*}>0, W^{u}(O) \cap\left\{z=z_{*}\right\}$ is a spiral curve which winds around the point $W^{u}(P) \cap\left\{z=z_{*}\right\}$.

It follows from Lemma 4 in the Appendix that the curves $W^{u}(O) \cap\left\{z=\lambda_{*}\right\}$ and $\left\{z=\lambda_{*}, x=0\right\}$ intersect an infinite number of times and that each of these intersections corresponds to distinct solutions of (2). This explains the oscillating behaviour of the bifurcation diagram; see Figure 5 in section 6 . The continuity of the solutions of the initial value problem in terms of the parameters implies that if $\lambda$ is close enough to $\lambda_{*}$, a large number of these intersections will persist. This concludes the proof.

\section{Proof of Theorem 3}

Here we consider the equation

$$
\left\{\begin{array}{l}
-\Delta u=\lambda(u+1)^{p}+\lambda(u+1)^{q} \text { in } B \\
u>0 \text { in } B, \quad u=0 \text { on } \partial B .
\end{array}\right.
$$

We are looking for radially symmetric solutions of this equation, namely solutions of the boundary value problem

$$
\begin{cases}u^{\prime \prime}+\frac{N-1}{r} u^{\prime}+\lambda(u+1)^{p}+\lambda(u+1)^{q}=0, & 0<r<1, \\ u^{\prime}(0)=0, \quad u(1)=0 \text { and } \quad u(r)>0 \text { for } \quad 0<r<1 .\end{cases}
$$

Let us denote $v=u+1$, so that this problem is equivalent to

$$
\left\{\begin{array}{l}
v^{\prime \prime}+\frac{N-1}{r} v^{\prime}+\lambda v^{p}+\lambda v^{q}=0, \quad 0<r<1 \\
v^{\prime}(0)=0, \quad v(1)=1 \text { and } \quad v(r)>1 \text { for } 0<r<1 .
\end{array}\right.
$$

We absorb the parameter $\lambda$ by setting $r=s / \sqrt{\lambda}, v(r)=w(s)$, so that the problem becomes

$$
\left\{\begin{array}{l}
w^{\prime \prime}+\frac{N-1}{s} w^{\prime}+w^{p}+w^{q}=0, \quad 0<r<1 \\
w^{\prime}(0)=0, \quad w(\sqrt{\lambda})=1 \text { and } \quad w(s)>1 \text { for } 0<s<\sqrt{\lambda} .
\end{array}\right.
$$

Similarly as before, we introduce for (14) the tranformation

$$
x(t)=\left.s^{\frac{2}{p-1}} w(s)\right|_{s=e^{t}}
$$

to obtain the equivalent equation

$$
x^{\prime \prime}+\alpha x^{\prime}+x^{p}+e^{\gamma t} x^{q}-\beta x=0, \quad-\infty<t<\nu,
$$

where $\nu=\ln (\sqrt{\lambda})$ and $\alpha, \beta, \gamma$ are explicit positive constants given by

$$
\alpha=(N-2)-\frac{4}{p-1}, \quad \beta=\frac{2}{p-1}\left(N-2-\frac{2}{p-1}\right), \quad \gamma=2 \frac{p-q}{p-1} .
$$

Let us observe that conditions on $w$ in (14) become

$$
x(t)>e^{\frac{2 t}{p-1}} \quad \text { in } \quad(-\infty, \nu), \quad \lim _{t \rightarrow-\infty} x(t) \rightarrow 0 \quad \text { and } \quad x(\nu)=\lambda^{\frac{1}{p-1}} .
$$


Introducing the variables $y=x^{\prime}$ and $z=e^{\gamma t}$ as in the proof of Theorem 10 we obtain the three-dimensional system

$$
\left\{\begin{array}{l}
x^{\prime}=y, \\
y^{\prime}=-\alpha y+\beta x-x^{p}-z x^{q}, \\
z^{\prime}=\gamma z
\end{array}\right.
$$

with as before the additional condition $z \geq 0$. A solution $x(t)$ of problem (15)-(16) is in correspondence with a solution $\varphi(t)=(x(t), y(t), z(t))$ of (17) such that

$$
x(t)>e^{\frac{2 t}{p-1}} \quad \text { in } \quad(-\infty, \nu), \quad \lim _{t \rightarrow-\infty} \varphi(t)=(0,0,0) \quad \text { and } \quad x(\nu)=\lambda^{\frac{1}{p-1}} .
$$

In terms of system (17) the condition $x(t)>e^{2 t /(p-1)}$ is then equivalent to $x>$ $z^{1 /(p-q)}$. In fact, $z=e^{\gamma t}$ and $\gamma=2 \frac{p-q}{p-1}$. We observe that the role of the surface given by $x=z^{1 /(p-q)}$ is the same as that of the plane $\{x=0\}$ in system (9), in the sense that trajectories which satisfy respectively (18) and (10) must be to the right of these surfaces.

On the other hand, the boundary condition $x(\nu)=\lambda^{1 /(p-1)}$ can be written as $x(\nu)=z^{1 /(p-q)}(\nu)$. This means that the corresponding solution of system (17) has to intersect the surface $x=z^{1 /(p-q)}$ when $t=\nu$.

Let us briefly explain the phase space structure of system (17) which in the end turns out to be analogous to that of system (9). We have two hyperbolic points of (17) which lie on the plane $\{z=0\}, O=(0,0,0)$ and $P=\left(\beta^{1 /(p-1)}, 0,0\right)$. The system restricted to $z=0$ is invariant under the associated flow and inside $\{z=0\}$; there is an orbit which connects the points $O$ and $P$. This orbit coincides with $W^{u}(O) \cap\{z=0\}$. On the other hand, if $p$ satisfies

$$
p<\frac{N-2 \sqrt{N-1}}{N-2 \sqrt{N-1}-4} \quad \text { or } \quad N \leq 10,
$$

we obtain that $P$ is a spiral attractor in $\{z=0\}$. Indeed, linearizing around this point, we get the eigenvalues

$$
\frac{1}{2}\left(-\alpha \pm \sqrt{\alpha^{2}-4 \beta(p-1)}\right)
$$

which are complex with non-zero imaginary part if and only relation (19) holds. The point $P$ is an attractor in $\{z=0\}$, since $\alpha>0$.

Now, for $z \geq 0$ the unstable manifold of $O, W^{u}(O)$, is two-dimensional. Linearizing around $O$, we obtain one negative eigenvalue and the two others are positive: $\gamma$ and $2 /(p-1)$, with respective eigenvectors $(0,0,1)$ and $(1,2 /(p-1), 0)$.

Linearizing around $P$, we have one positive eigenvalue $\gamma$, so that the unstable manifold of $P, W^{u}(P)$, is one-dimensional. Its associated eigenvector is $(1, \gamma$, $\left.\frac{(p-1) \beta+2 \alpha+\gamma^{2}}{\beta^{1 /(p-1)}}\right)$. The stable manifold of $P, W^{s}(P)$, is two-dimensional and lies on $z=0$. Moreover, the heteroclinic orbit which connects $O$ and $P$ is contained in $W^{s}(P)$. This discussion can be summarized as follows.

Lemma 3. Consider system (17). The following assertions hold:

(i) The unstable manifold of $P, W^{u}(P)$, corresponds to the positive singular solution of (13). This solution is unique since $W^{u}(P)$ is one dimensional.

(ii) $W^{u}(P)$ lies on the boundary of $W^{u}(O)$. 
(iii) Solutions of system (17)-(18) lie inside of $W^{u}(O)$. Moreover, they must be to the right of the surface $x=z^{1 /(p-q)}$ and eventually cross it. The unstable manifold of $P$ crosses this surface at a some first value $z=\lambda_{*}>0$.

(iv) The condition $x(\nu)=\lambda^{1 /(p-1)}$ in (18), in terms of the $W^{u}(O)$, means that this manifold intersects the surface $x=z^{1 /(p-q)}$.

Assertions (i) and (ii) have already been discussed for system (9). Assertion (iv) directly follows from conditions (18). Let us now observe that a solution $u(r)$ of (13) which for some $r_{0}>0$ satisfies $u\left(r_{0}\right)>0$, also satisfies

$$
-\left(r^{N-1} u^{\prime}(r)\right)^{\prime} \geq 2 \lambda r^{N-1}
$$

in any interval $\left(r_{0}, r_{1}\right)$ where $u(r)>0$. It readily follows that there must exist a first $r_{1}>r_{0}$ with $u\left(r_{1}\right)=0$. The condition $u=0$ in terms of system (17) is nothing but the surface $\left\{z=x^{p-1}\right\}$. A consequence is that $W^{u}(O) \cap\left\{z=x^{p-1}\right\}$ is a curve in a neighborhood of $O$, which locally splits $W^{u}(O)$ into two components. Trajectories which lie below this surface are eventually forced to cross it. This of course also holds for a trajectory representing the unstable manifold of $P$. The validity of (iii) thus follows.

Let us conclude the proof of Theorem 2. The value $z=\lambda_{*}$ represents exactly the value for which the unique trajectory corresponding to the singular solution crosses the surface defined by the zero boundary value for the solution of (13). The rest of the theorem follows in exactly the same way as in the proof of Theorem 1 .

\section{Appendix: Some topological facts}

This Appendix is devoted to the proofs of Lemmas 1 and 2, and to the statement (see below) and the proof of Lemma 4 which enters in the proof of Theorems 1 and 3 as follows. We have to prove that the two pairs of curves

$$
\begin{array}{ll}
W^{u}(O) \cap\left\{z=\lambda_{*}\right\} & \text { and } \quad\left\{z=\lambda_{*}, x=0\right\}, \\
W^{u}(O) \cap\left\{z=\lambda_{*}\right\} & \text { and } \quad\left\{z=\lambda_{*}, x=z^{1 /(p-q)}\right\},
\end{array}
$$

intersect infinitely many times. $W^{u}(O) \cap\left\{z=\lambda_{*}\right\}$ is a spiral curve which winds around the point given by $W^{u}(P) \cap\left\{z=\lambda_{*}\right\}$. By definition of $\lambda_{*}$, we also know that this point lies on the line $\left\{z=\lambda_{*}, x=0\right\}$ or on the curve $\left\{z=\lambda_{*}, x=z^{1 /(p-q)}\right\}$, in the case of Theorems 1 and 3 , respectively. The result is going to be a consequence of a simple topological lemma.

Let $P_{*}$ be a point in the plane and consider a spiral curve $\sigma(t)$ around $P_{*}$ of the following form:

$$
\sigma(t)=P_{*}+r(t) e^{i \theta(t)}, \quad t \in[0,+\infty)
$$

where $r>0$ and $\theta$ are continuous functions in $[0,+\infty)$, such that $r(t) \rightarrow 0$ as $t \rightarrow+\infty$ and $\theta(t) \rightarrow-\infty$ as $t \rightarrow+\infty$. We can assume that $0<r(t)<r(0)$ for $t>0$, and also that $\theta(t) \leq \theta(0)=0$ for $t \geq 0$.

Now let $\tau(s), s \in[0,1]$, be a continuous curve, such that $\tau(0)=P_{*}$, and assume additionally that $\tau(s)$ is to the right of $P_{*}$ for $s \in(0,1]$. Writing

$$
\tau(s)=P_{*}+\rho(s) e^{i \mu(s)}, \quad s \in(0,1]
$$

where $\rho>0$ and $\mu$ are continuous functions in $(0,1]$, means that $\rho(0)=0$ and that there exists an integer $k \in \mathbb{Z}$ such that $-\pi / 2 \leq \mu-2 k \pi \leq \pi / 2$. Assume further that both $\sigma$ and $\tau$ do not have self-intersections. 
Lemma 4. Let $\sigma$ and $\tau$ be curves as above. Assume that $\rho(s)<r(0)$ for all $s \in(0,1]$. Then the curves $\sigma$ and $\tau$ intersect an infinite number of times.

Proof of Lemma 4. In polar coordinates centered around $P_{*}$, liftings of $\sigma$ and $\tau$ are respectively given by $\tilde{\sigma}(t)=(r(t), \theta(t))$ and $\tilde{\tau}(s)=(\rho(s), \mu(s))$. Jordan's theorem implies that the curve $\tilde{\sigma}$ separates the strip $(0, r(0)] \times \mathbb{R}$ of the $r-\theta$ plane into two components $A_{-}$and $A_{+}$to the left and to the right of the curve $\tilde{\sigma}$, respectively. Also, the curve $\tilde{\tau}$ entirely lies inside this strip. The family of translates $\tilde{\tau}_{k}=$ $\tilde{\tau}+(0,-2 k \pi)$ are also liftings of $\tau$. Given a number $n$, consider $t_{n}$ such that $\theta(t)<\theta\left(t_{n}\right)$ and $r(t)<1 / n$ for all $t>t_{n}$. Then, if $k$ is chosen sufficiently large, the following happens: there are points of $\tilde{\tau}_{k}$ which lie on $A^{-}$, while necessarily $\tilde{\tau}_{k}(1)$ lies on $A^{+}$. It follows by connectedness that the curve $\tilde{\tau}_{k}$ intersects $\tilde{\sigma}$. Since the $r$-coordinate of this point is less than $1 / n$, and $n$ is arbitrary, infinitely many intersections of the original curves $\sigma$ and $\tau$ are obtained, as desired.

Proof of Lemma 1. For the proof of this result we will make use of the well-known Palis $\lambda$-lemma; see for instance [28, or [18. Its statement in the context we are dealing with is as follows.

Lemma 5. Consider in $\mathbb{R}^{n}$ an ODE of the form $x^{\prime}=f(x)$, where $f$ is of class $C^{1}$, in a neighborhood $\mathcal{V}$ of a hyperbolic singularity $P$. Let $X_{t}$ denote its associated flow and consider the (local) stable and unstable manifolds $W^{s}(P), W^{u}(P)$, with respective dimensions $n_{s}$ and $n_{u}$. Let $D$ be an $n_{u}$-dimensional disk which intersects transversally $W^{s}(P)$ and contains a point $Q$ of $W^{u}(P)$. Let $B^{u}$ be any disk inside $W^{u}(P)$ which contains $Q$. Let $D_{t}$ be the connected component of $X_{t}(D) \cap \mathcal{V}$ which contains $X_{t}(Q)$. Then given $\varepsilon>0$, there exists a $t_{0}>0$ such that $D_{t}$ is $C^{1} \varepsilon$-close to $B^{u}$ for all $t>t_{0}$. In particular, given any point $Q^{\prime} \in B^{u}$, there is a point in $D_{t}$ which is at a distance less than $\varepsilon$ from $Q^{\prime}$.

As we have seen, $P$ is a hyperbolic singularity whose unstable manifold $W^{u}(P)$ is a one-dimensional curve, and $W^{s}(P)$ two-dimensional. Take a short segment transversal to the plane $\{z=0\}$ which lies entirely in the two-dimensional manifold $W^{u}(O)$ (take it for instance close and almost parallel to the $z$-axis). By virtue of the above lemma, the flow will take this segment into a one-dimensional segment, still contained in $W^{u}(O)$, which gets arbitrarily uniformly close to any given finite piece of the curve $W^{u}(P)$. This proves that $W^{u}(P)$ lies in the boundary of $W^{u}(O)$.

Proof of Lemma 2. To this end we employ the following result, due to Belickir [3, 4].

Lemma 6. Consider an ODE of the form $x^{\prime}=f(x)$ with $f\left(x_{0}\right)=0$ and $f$ smooth in a neighborhood of $x_{0}$. Assume that $x_{0}$ is a hyperbolic saddle of $f$, with eigenvalues $\lambda_{1}, \ldots, \lambda_{n}$. Assume also that none of the relations $\operatorname{Re} \lambda_{i}=\operatorname{Re} \lambda_{j}+\operatorname{Re} \lambda_{k}$ is fulfilled. Then the system is $C^{1}$-equivalent in a neighborhood of $x_{0}$ to its linear part, in the sense that there is a $C^{1}$ diffeomorphism $\Phi$ from a neighborhood of $x_{0}$ onto a neighborhood of 0 , with $\Phi\left(x_{0}\right)=0$ which takes trajectories of the system $x^{\prime}=f(x)$ into trajectories of the linear system $y^{\prime}=f^{\prime}\left(x_{0}\right) y$, preserving orientation.

This result applies immediately to systems (9) and (17) around $P$ if the unstable eigenvalues are not real, since we have two eigenvalues with the same negative real parts, and a third eigenvalue which is positive. Then none of the relations $\operatorname{Re} \lambda_{i}=\operatorname{Re} \lambda_{j}+\operatorname{Re} \lambda_{k}$ is possible. 


\section{Figures}

In this section, computations are done in the case $N=5$ and $p=7 / 3+0.3$.

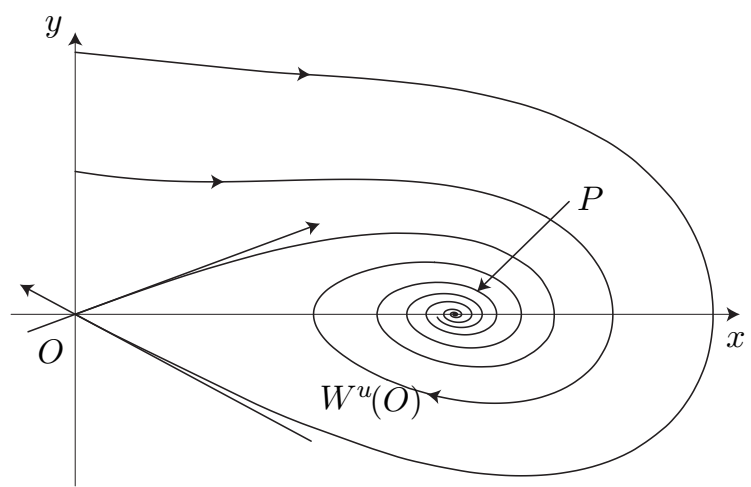

Figure 1. The phase space restricted to $\{z=0\}$ and the heteroclinic trajectory $t \mapsto \psi(t)$ connecting $O$ to $P$ in this plane.

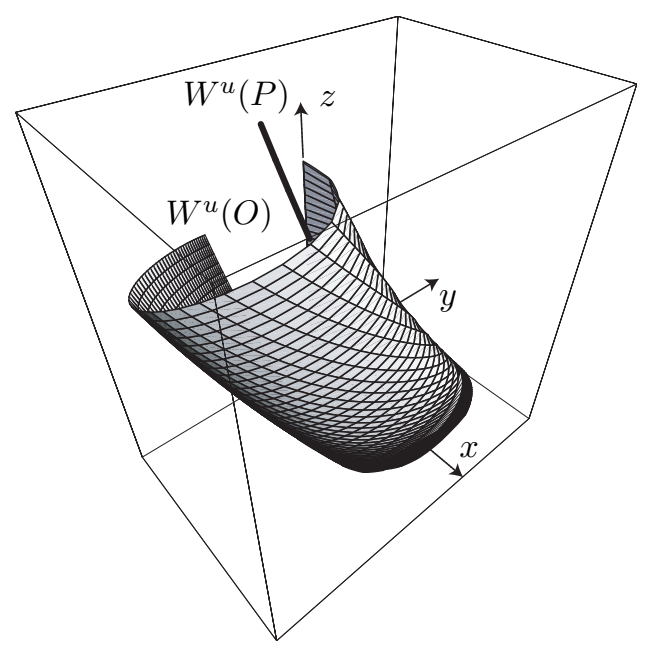

Figure 2. The 3-dimensional phase space with the 2-dimensional unstable manifold $W^{u}(O)$, which contains the axis $\{x=0, y=0\}$, and the 1-dimensional unstable manifold $W^{u}(P)$, which is contained in the adherence of $W^{u}(O)$. 

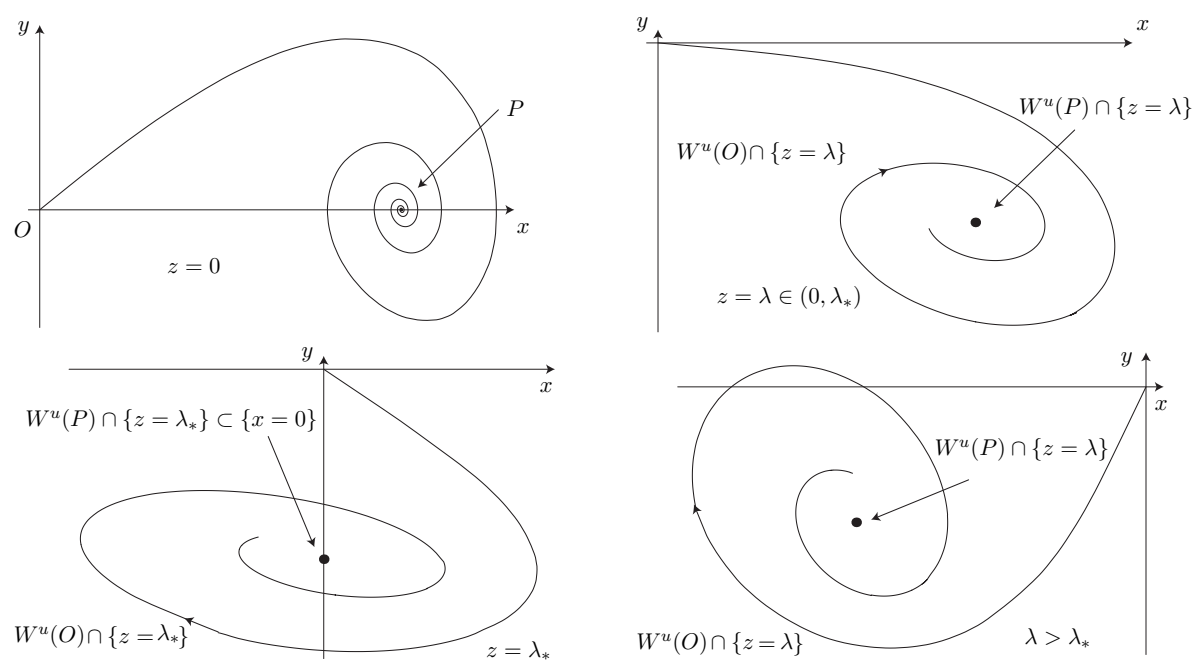

FiguRE 3. Intersection of the unstable manifolds $W^{u}(O)$ and $W^{u}(P)$ with planes $\left\{z=z_{*}\right\}$ for $z_{*}=0, z_{*}=\lambda \in\left(0, \lambda_{*}\right), z_{*}=\lambda_{*}$, $z_{*}=\lambda>\lambda_{*}$.

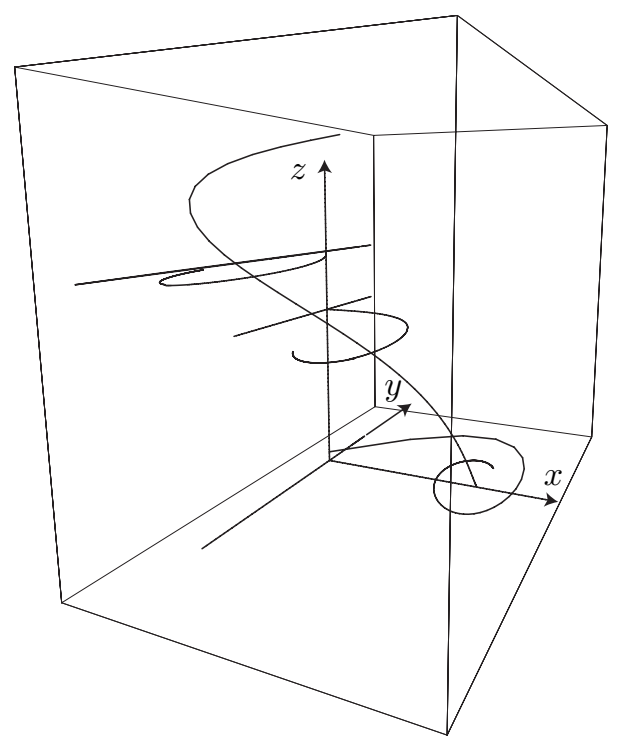

Figure 4. Several 3-dimensional sections of the unstable manifold $W^{u}(O)$ corresponding to the planes $\left\{z=z_{*}\right\}$ for $z_{*}=\lambda \in\left(0, \lambda_{*}\right)$ and the 3-dimensional representation of $W^{u}(P)$. 


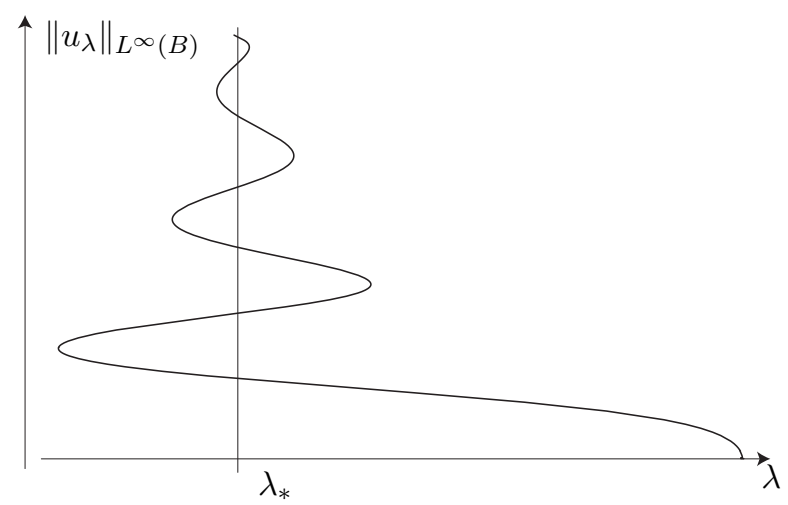

Figure 5. The bifurcation diagram in $\mathbb{R}^{+} \times L^{\infty}(B)$.

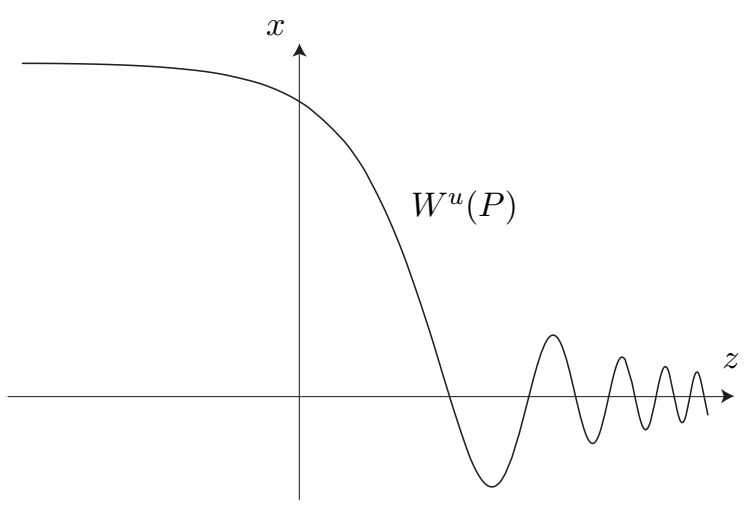

Figure 6. The unstable manifold $W^{u}(P)$ in $(z, x)$ coordinates ( $z$ is shown in a logarithmic scale). Finding the first zero determines $z_{*}=\lambda_{*}$. Here for $N=5$ and $p=7 / 3+0.3$, we find $\lambda_{*} \approx 12.07 \ldots$

\section{ACKNOWLEDGEMENTS}

This work has been partly supported by FONDECYT grant 1050968 (I.F.), and by ECOS-Conicyt under contracts C98E03 and C02E08. The authors thank the referee for a useful question which let them clarify a point in the proof of Theorem1.

\section{REFERENCES}

[1] F.V. Atkinson, H. Brezis, H., L.A. Peletier, Nodal solutions of elliptic equations with critical Sobolev exponents, J. Differential Equations 85 (1990), no. 1, 151-170. MR1052332 (91e:35021)

[2] R. Bamon, I. Flores, M. del Pino, Ground states of semilinear elliptic equations: a geometric approach, Ann. Inst. H. Poincaré Anal. Non Linéaire 17 (2000), 551-581. MR1791878 (2001k:35082)

[3] G.R. Belickiŭ, Equivalence and normal forms of germs of smooth mappings, Russ. Math. Surv. 33 (1978), 101-177. MR0490708 (80k:58017) 
[4] G.R. Belickiǔ, Normal forms, invariants and local mappings, Naukova Dumka, Kiev, 176 (1979). MR0537763 (81h:58014)

[5] R. Benguria, J. Dolbeault, M.J. Esteban, Classification of the solutions of semilinear elliptic problems in a ball, J. Differential Equations 167 (2000), no. 2, 438-466. MR1793200 (2001k:35083)

[6] H. Brezis, L. Nirenberg, Positive solutions of nonlinear elliptic equations involving critical Sobolev exponents, Comm. Pure and Appl. Math. 36 (1983) no. 4, 437-477. MR0709644 (84h:35059)

[7] C. Budd, J. Norbury, Semilinear elliptic equations and supercritical growth, J. Differential Equations 68 (1987) no. 2, 169-197. MR0892022 (88i:35056)

[8] C.B. Clemons, C.K.R.T. Jones, A geometric proof of the Kwong-McLeod uniqueness result, SIAM J. Math. Anal. 24 (1993), 436-443. MR.1205535 (94f:35047)

[9] G. Cerami, S. Solimini, M. Struwe, Some existence results for superlinear elliptic boundary value problems involving critical exponents, J. Funct. Anal. 69 (1986), no. 3, 289-306. MR.0867663 (88b:35074)

[10] M. Del Pino, J. Dolbeault, M. Musso, "Bubble-tower" radial solutions in the slightly supercritical Brezis-Nirenberg problem, J. Differential Equations 193 (2003), no. 2, 280-306. MR1998635 (2004h:35077)

[11] M. Del Pino, J. Dolbeault, M. Musso, A phase plane analysis of the "Multi-bubbling" phenomenon in some slightly supercritical equations, Monatsh. Math. 142 (2004), no. 1-2, 57-79. MR2065022 (2005h:35103)

[12] M. Del Pino, J. Dolbeault, M. Musso, The Brezis-Nirenberg problem near criticality in dimension 3, J. Math. Pures Appl. (9) 83 (2004), no. 12, 1405-1456. MR2103187|(2005h:35123)

[13] J. Dolbeault, M. Esteban, M. Ramaswamy, Radial singular solutions of a critical problem in a ball, Differential Integral Equations 15 (2002), no. 12, 1459-1474. MR1920255(2003f:35102)

[14] L. Erbe, M. Tang, Uniqueness of positive radial solutions of $\Delta u+K(|x|) \gamma(u)=0$, Differential Integral Equations 11 (1998), no. 4, 663-678. MR1666214 (99j:35046)

[15] L. Erbe, M. Tang, Uniqueness theorems for positive radial solutions of quasilinear elliptic equations in a ball, J. Differential Equations 138 (1997), no. 2, 351-379. MR1462272 (98k:35069)

[16] R. Fowler, Further studies of Emden's and similar differential equations, Quart. J. Math. 2 (1931), 259-288.

[17] B. Gidas, W.-M. Ni, L. Nirenberg, Symmetry and related properties via the Maximum Principle, Commun. Math. Phys. 68 (1979), 209-243. MR0544879 (80h:35043)

[18] M. Hirsch, C. Pugh, M. Schub, Invariant Manifolds, Lecture Notes in Math. 583, SpringerVerlag, New York (1977). MR0501173 (58:18595)

[19] M.K. Kwong, Y. Li, Uniqueness of radial solutions of semilinear elliptic equations, Trans. A.M.S. 333 (1992), 339-363. MR1088021 (92k:35102)

[20] J. Jacobsen, K. Schmitt, The Liouville-Bratu-Gelfand problem for radial operators, J. Differential Equations 184 (2002), 283-298. MR1929156 (2003g:34036)

[21] R. Johnson, X.-B. Pan, Y. Yi, The Melnikov method and elliptic equations with critical exponent, Indiana Univ. Math. J. 43 (1994), 1045-1077. MR.1305959(95k:35068)

[22] D.D. Joseph, T.S. Lundgren, Quasilinear Dirichlet problems driven by positive sources, Arch. Rational Mech. Anal. 49 (1972/73), 241-269. MR0340701 (49:5452)

[23] F. Merle, L.A. Peletier, Positive solutions of elliptic equations involving supercritical growth, Proc. Roy. Soc. Edinburgh Sect. A 118 (1991), no. 1-2, 49-62. MR1113842 (92g:35074)

[24] F. Merle, L.A. Peletier, J. Serrin, A bifurcation problem at a singular limit, Indiana Univ. Math. J. 43 (1994), no. 2, 585-609. MR 1291530 (95j:35024)

[25] W.-M. Ni, Uniqueness of solutions of nonlinear Dirichlet problems, J. Differential Equations 50 (1983), no. 2, 289-304. MR0719451 (85i:35060)

[26] T. Ouyang, J. Shi, A bifurcation approach to the exact multiplicity of positive solutions of $\Delta u+f(u)=0$ on the unit ball, Dynamical systems and differential equations, Vol. II (Springfield, MO, 1996). MR1721186 (2000j:35105)

[27] T. Ouyang, J. Shi, Exact multiplicity of positive solutions for a class of semilinear problem. II, J. Differential Equations 158 (1999), no. 1, 94-151. MR1721723 (2001b:35117)

[28] J. Palis, W. de Melo, Geometric theory of dynamical systems: an introduction, SpringerVerlag, New York, Heidelberg, Berlin (1982). MR0669541 (84a:58004) 
[29] S.I. Pohozaev, On the eigenfunctions of the equation $\Delta u+\lambda f(u)=0$, Dokl. Akad. Nauk SSSR 165 (1965), 36-39. MR0192184 (33:411)

[30] J. Serrin, M. Tang, Uniqueness of ground states for quasilinear elliptic equations, Indiana Univ. Math. J. 49 (2000), no. 3, 897-923. MR1803216 (2002d:35072)

[31] J. Shi, Exact multiplicity of positive solutions to superlinear problems, Elec. Jour. Diff. Equa. Conf. 10 (2002), 257-265. MR.1976648 (2004d:35079)

[32] P.N. Srikanth, Uniqueness of solutions of nonlinear Dirichlet problems, Differential Integral Equations 6 (1993), no. 3, 663-670. MR.1202564 (94b:35122)

[33] L.-Q. Zhang, Uniqueness of positive solutions to semilinear elliptic equations. (Chinese), Acta Math. Sci. (Chinese) 11 (1991), no. 2, 130-142. MR.1129746 (92j:35055)

[34] L.-Q. Zhang, Uniqueness of positive solutions of $\Delta+u+u^{p}=0$ in a ball, Comm. Partial Differential Equations 17 (1992), nos. 7-8, 1141-1164. MR1179281 (94b:35125)

Ceremade (UmR CNRS no. 7534), Université Paris IX-Dauphine, Place de Lattre de Tassigny, 75775 Paris Cédex 16, France

E-mail address: dolbeaul@ceremade.dauphine.fr

Departamento de Ciencias Básicas, Facultad de Ciencias, Universidad del Bío-Bío, Casilla 447, Chillán, Chile

Current address: Departamento de Ingeniería Matemática, Universidad de Chile, Casilla 170, Coreo 3, Santiago, Chile 